\title{
How to look for planetary transits using small telescopes and commercial CCDs in developing countries
}

\author{
Eder Martioli ${ }^{1}$ and F. Jablonski ${ }^{1}$ \\ ${ }^{1}$ Divisão de Astrofísica, Instituto Nacional de Pesquisas Espaciais, \\ São José dos Campos, Brazil \\ email: eder@das.inpe.br, chico@das.inpe.br
}

\begin{abstract}
The main goal of this work is to have a better understanding of the problems and characteristics of photometric surveys with small-sized affordable equipment, like the one available at the Astrophysics Division/INPE, in São José dos Campos, Brazil. The use of low-cost instruments is appealing in the context of the detection of Extrasolar Planets (ESP), in the sense that many observers are available for survey and follow-up programmes. It could also make possible the inclusion of many developing countries in the search for planetary transits. We describe the data collection and analysis procedure for differential photometry of the transit of HD 209458 b, using a small telescope and a commercial CCD camera. According to the HST observations of Brown et al. (2001), the transit produces a box-shaped light curve with $2 \%$ depth and 184-min duration. The orbital period is $\sim 3.5$ days. The equipment consists of a f/10, 11" Schmidt-Cassegrain Celestron telescope equipped with a SBIG ST7E CCD camera. Since the seeing at the campus is quite poor, we used a focal reducer to produce an effective focal ratio of about $\mathrm{f} / 5$, still keeping a good sampling of the PSF but with a larger field of view. The larger field of view allows the simultaneous observation of a relatively bright nearby star, suitable for differential photometry. We discuss the IRAF reduction procedures for the large number of images collected and present the results obtained in the transit of September 8, 2004
\end{abstract}

Keywords. techniques: photometric, stars: planetary systems, instrumentation: miscellaneous

\section{Introduction}

HD 209458 is one of the stars known to bear an Extra-Solar Planet (ESP). The detection of this planet was firstly done by Henry et al. (2000) using the conventional radial-velocity technique. Subsequently, Charbonneau et al. (2000) showed that a photometric transit can be detected in the system. The detection of photometric transits requires high-quality data since the decrease in flux is only $2 \%$ and the event spans only 3.5 hours.

The probability of a transit to happen is a few per cent, and thus many ESP could be detected photometrically. However, the difficulties in achieving the required photometric precision from ground-based observatories has kept the number of detections small.

This scenario is beginning to change with space instruments like MOST and COROT which are able to reach photometric precisions of micro-magnitudes. Similarly, groundbased photometric surveys of a large number of targets would also be effective in detecting transits. The OGLE survey, even though designed for different purposes, has detected tens of candidate planetary transits. 


\section{Equipment}

Table 1 summarizes the instrumentation and Figure 1 shows the 11" telescope used in our work.

Table 1. Description of Instrumentation

\begin{tabular}{lc}
\hline Telescope & Celestron 11" Schmidt-Cassegrain \\
Accessories & focal reducer \\
Effective focal ratio & f $/ 5$ \\
Filters & clear \\
Mount & equatorial Losmandi G11 \\
CCD & SBIG ST7E \\
\hline
\end{tabular}

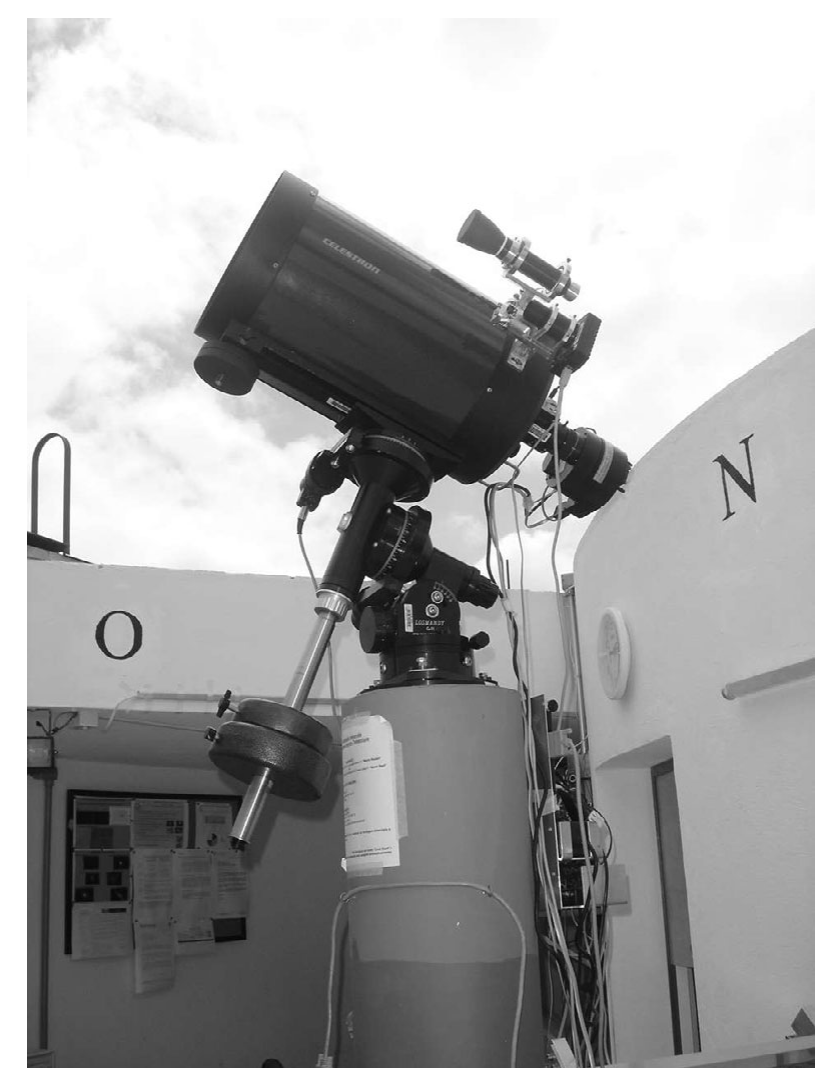

Figure 1. The 11" telescope in its mount at the main campus of INPE.

\section{Data management}

\subsection{Data acquisition}

The images were collected using the CCDSoft (version 5.00.126) software. Since HD 209458 is quite bright we used 3 -sec integrations to be safely under the saturation limit. We added a 10-sec wait-time to the observing cycle to keep the number of images small. The CCD temperature was kept close to $-10^{\circ} \mathrm{C}$ during the observing run. Since we did not use auto-guiding, small corrections were done manually to keep the objects more or 


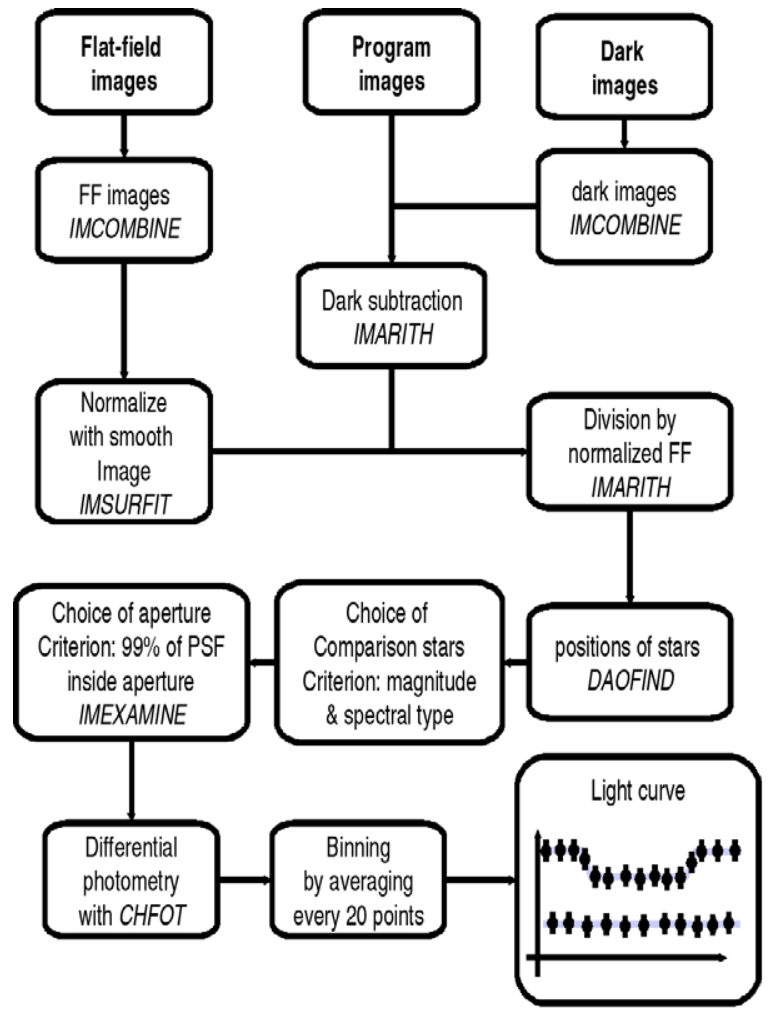

Figure 2. Flowchart showing the steps to be followed in the data reduction.

less in the same region of the detector. This is important since the focal reducer used to increase the field-of-view has strong vignetting as one goes to the borders of the field.

The usual flat-field images were taken to allow a pixel-to-pixel sensitivity correction to be carried out. Also, dark frames with the same integration time as the programme images were collected to allow the subtraction of the dark noise component.

The measurements were started one hour before the beginning of transit and lasted until the end of the event, resulting in a total of 895 CCD frames. Unfortunately, the sky conditions at the end of the event were quite poor.

\subsection{Data reduction}

The preparation of the data was carried out under IRAF and consisted of: (i) producing a median dark frame with imcombine; (ii) flat-field median frame from which the dark frame was subtracted; (iii) normalized flat-field from the division of combined flat-field frame from step (ii) by a smooth image fit to the same image with imsurfit. The $R M S$ noise of the normalized flat-field frame is $0.2 \%$. This can be considered quite good for a low-cost CCD detector.

The flux extraction was done with an IRAF script called chfot. This task automatically identifies the stars in each frame, centers precisely in each object, extracts the correspondent fluxes, calculates the magnitude differences and presents the data together with the heliocentric Julian date. A useful feature of the program is its capability to add up all the comparison stars. This is important in cases (like the present one) where the variable object is the brightest in the field-of-view. 


\section{Results}

The original light curves that produced the binned version in Figure 3 have $R M S$ errors comparable to the depth of the transit itself, namely, $2 \%$. This makes it difficult to visualize the event. To improve visualization we combined the original points averaging them in bins containing 20 points. A side benefit of the binning operation is to get a better idea of the absolute precision of the measurements.
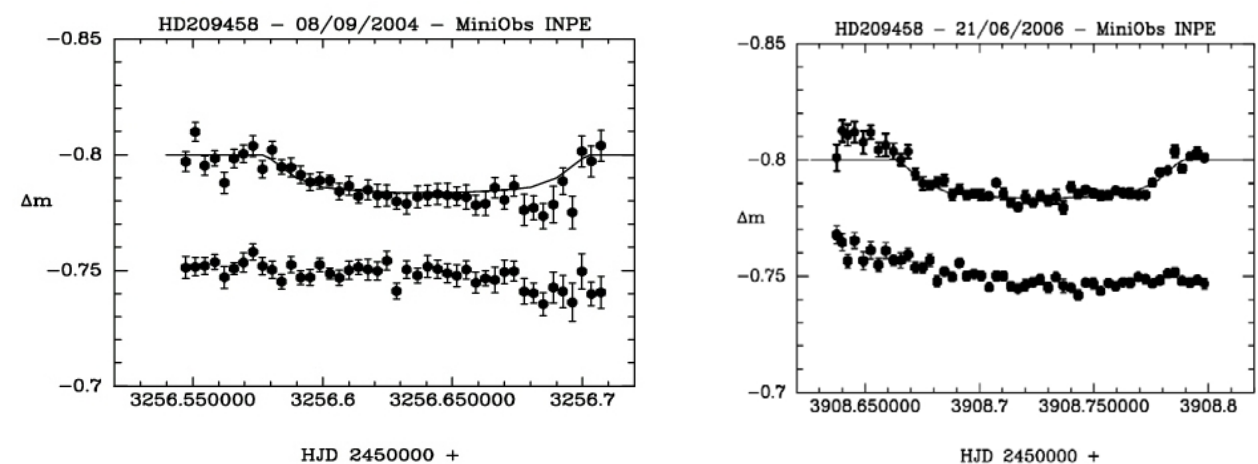

Figure 3. Light curves of the transit of HD 209458 b (top) and the sum of comparison stars (bottom) obtained in 2004 September 08 (left) and 2006 June 21st (right).

Figure 3 shows the binned light curves for HD 209458 and for the sum of the comparison stars. We superimposed the light curve of the HST data from Brown et al. (2001). One can see that for the two transits measured there is consistency in terms of time of beginning of the transit, its depth and duration. The photometric conditions were poorer in 2004 September 08 at the end of the run. The opposite happened in 2006 June 21st. The light curves show that there are limits even for a robust technique like differential photometry. Besides the larger extinction itself, differential extinction and differential refraction contribute to systematic trends and larger scatter in the measurements as one goes down to the horizon.

Some possible improvements given the lessons learned with the acquisition, reduction and analysis of the data can be summarized as:

- To use a larger unvignetted field-of-view to obtain more comparison stars.

- To use auto-guiding to keep the target in the same (good) area of the detector.

- To use a red bandpass filter to minimize the effects of differential extinction and differential refraction.

- To select comparison stars as close in spectral type to HD 209458 as possible.

\section{Acknowledgements}

IRAF is distributed by the National Optical Astronomy Observatories, which are operated by the Association of Universities for Research in Astronomy, Inc., under cooperative agreement with the National Science Foundation.

\section{References}

Charbonneau, D., Brown, T.M., Latham, D.W., Mayor, M. 2000, ApJL 529, L45-L48

Brown, T.M., Charbonneau, D., Gilliland, R.L., Noyes, R.W., Burrows, A. 2001, ApJ 552, 699

Henry, G.W., Marcy, G.W., Butler, R.P., Vogt, S.S. 2000, ApJL 529, L41-L44 